\title{
Comparison of Obesity Indices and Triglyceride Glucose Related Parameters to Predict Type 2 Diabetes Mellitus Among Normal-Weight Elderly in China
}

\section{Pan Ke}

Huazhong University of Science and Technology Tongji Medical College School of Public Health

\section{Xia Wu}

Baoan Distract Songgang People's Hospital

\section{Minzhi Xu}

Huazhong University of Science and Technology Tongji Medical College School of Public Health Jie Feng

Huazhong University of Science and Technology Tongji Medical College School of Public Health Hongbin Xu

Huazhong University of Science and Technology Tongji Medical College School of Public Health

\section{Yong Gan}

Huazhong University of Science and Technology Tongji Medical College School of Public Health

\section{Chao Wang}

Huazhong University of Science and Technology Tongji Medical College School of Public Health

\section{Zhenyu Deng}

Baoan Distract Songgang People's Hospital

\section{Xiang Liu}

Baoan Distract Songgang People's Hospital

\section{Wenning Fu}

School of Nursing,Tongji Medical College,Huazhong University of Science and Techology

\section{Qingfeng Tian}

School of Public Health,Zhengzhou University

\section{Yan He}

School of Public Health,Zhengzhou University

\section{Lirong Zhong}

School of Public Health,Hubei University of Medicine

\section{Heng Jiang}

Center for Alcohol Policy Research,School of Psychology and Public Health,La Trobe University

\section{Zuxun Lu ( $\nabla$ zuxunlu@yahoo.com )}

Huazhong University of Science and Technology Tongji Medical College https://orcid.org/0000-00018432-5109 


\section{Original investigation}

Keywords: Normal-weight, Obesity Indices, TyG-related parameters, Type 2 diabetes mellitus (T2DM), Indicator

Posted Date: March 4th, 2021

DOI: https://doi.org/10.21203/rs.3.rs-263192/v1

License: (c) (i) This work is licensed under a Creative Commons Attribution 4.0 International License. Read Full License 


\section{Abstract}

Background : Although a significant proportion of type 2 diabetes mellitus (T2DM) cases arose from normal-weight individuals, studies on indicators that can predict T2DM in normal-weight people are limited. Accordingly, this study aims to investigate the predictive value of obesity indices and triglyceride glucose related parameters (TyG-related parameters) in T2DM among normal-weight Chinese elderly.

Methods : A total of 24,215 normal-weight Chinese elderly (age $\geq 60$ years) [body mass index-BMI (18.5$23.9 \mathrm{~kg} / \mathrm{m} 2$ )] were included. Obesity indices and triglyceride glucose related parameters (TyG-related parameters) included waist circumference (WC), waist-to-height ratio (WHtR), visceral adiposity index (VAl), lipid accumulation product (LAP), and TyG-related parameters (TyG, TyG-BMI, TyG-WC, and TyG-WHtR). Multivariate logistic regression analysis was performed to examine the associations between obesity-and TyG-related indices and T2DM. The areas under the curve (AUC) of the receiver-operating characteristic (ROC) curve analyses were used to evaluate and compare the predictive value of the different indices.

Results : The prevalence of T2DM was $14.2 \%$ in normal-weight individuals. Among the indices, TyG was significantly associated with T2DM among men and women, respectively (adjusted odds ratio-aOR 19.005; 95\% Cl 13.235-27.291) and (aOR 25.262; 95\% Cl 16.957-37.634). Compared with other indices, TyG had the highest AUC value for T2DM in men (AUC: $0.818,95 \% \mathrm{Cl} 0.810-0.825$ ) and women (AUC: $0.824,95 \% \mathrm{Cl}$ 0.814-0.833).

Conclusions : TyG is an effective marker and outperforms than other indices when predicting T2DM in the normal-weight Chinese elderly population.

\section{Full Text}

This preprint is available for download as a PDF.

\section{Tables}

Table 1 Demographic and clinical characteristics of the participants 


\begin{tabular}{|c|c|c|c|c|}
\hline & $\begin{array}{l}\text { Without } \\
\text { T2DM }(n=20,795)\end{array}$ & T2DM $(n=3420)$ & Total $(n=24,215)$ & $p$-value \\
\hline & $\begin{array}{l}\text { Median } \pm \text { SD or } \\
n(\%)\end{array}$ & \multicolumn{2}{|c|}{$\begin{array}{l}\text { Median } \pm S D \text { or } \\
n(\%)\end{array}$} & D or \\
\hline Sex & & & & $\nabla 0.001$ \\
\hline Men & $9281(38.33)$ & 1380(5.70) & $10,661(44.03)$ & \\
\hline Women & $11,514(47.55)$ & 2040(8.42) & $13,554(55.97)$ & \\
\hline Mean age (years) & $70.95 \pm 5.71$ & $71.47 \pm 5.70$ & $71.03 \pm 5.71$ & $\varangle 0.001$ \\
\hline Age category & & & & $\varangle 0.001$ \\
\hline $60-69$ yrs & $10,775(44.50)$ & 1596(6.59) & $12,371 \rrbracket 51.09 \rrbracket$ & \\
\hline $70-79$ yrs & $8021(33.12)$ & $1459(6.03)$ & $9480 \rrbracket 39.15 \rrbracket$ & \\
\hline 80 yrs+ & 1999(8.25) & $365(1.51)$ & $2364 \rrbracket 9.76 \rrbracket$ & \\
\hline BMI $\left(\mathrm{kg} / \mathrm{m}^{2}\right)$ & $21.75 \pm 1.44$ & $21.96 \pm 1.40$ & $21.78 \pm 1.44$ & $\varangle 0.001$ \\
\hline $\mathrm{SBP}(\mathrm{mm} \mathrm{Hg})$ & $132.87 \pm 18.57$ & $136.49 \pm 19.05$ & $133.38 \pm 18.68$ & $\varangle 0.001$ \\
\hline DBP $(\mathrm{mm} \mathrm{Hg})$ & $76.67 \pm 10.73$ & $77.11 \pm 10.57$ & $76.73 \pm 10.70$ & $\otimes 0.001$ \\
\hline $\mathrm{TC}(\mathrm{mmol} / \mathrm{L})$ & $5.13 \pm 1.11$ & $5.20 \pm 1.22$ & $5.14 \pm 1.13$ & $\otimes 0.001$ \\
\hline TG (mmol/L) & $1.45 \pm 0.97$ & $1.87 \pm 1.36$ & $1.51 \pm 1.04$ & 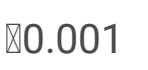 \\
\hline HDL-C (mmol/L) & $1.45 \pm 0.44$ & $1.37 \pm 0.40$ & $1.43 \pm 0.43$ & $\otimes 0.001$ \\
\hline LDL-C (mmol/L) & $3.01 \pm 0.95$ & $3.05 \pm 1.01$ & $3.02 \pm 0.96$ & $\nabla 0.001$ \\
\hline FPG (mmol/L) & $5.41 \pm 0.67$ & $9.28 \pm 2.69$ & $5.96 \pm 1.80$ & $\varangle 0.001$ \\
\hline \multicolumn{5}{|l|}{ Obesity indices } \\
\hline WC (cm) & $81.26 \pm 6.27$ & $82.73 \pm 6.16$ & $81.47 \pm 6.27$ & $\varangle 0.001$ \\
\hline WHtR & $0.51 \pm 0.04$ & $0.52 \pm 0.04$ & $0.51 \pm 0.041$ & $\nabla 0.001$ \\
\hline VAI & $1.91 \pm 3.01$ & $2.65 \pm 2.64$ & $2.02 \pm 2.97$ & $\otimes 0.001$ \\
\hline LAP & $30.27 \pm 25.21$ & $41.92 \pm 34.74$ & $31.91 \pm 27.08$ & $\nabla 0.001$ \\
\hline \multicolumn{5}{|l|}{$\begin{array}{l}\text { TyG-related } \\
\text { parameters }\end{array}$} \\
\hline TyG & $7.00 \pm 0.53$ & $7.74 \pm 0.63$ & $7.10 \pm 0.60$ & $\nabla 0.001$ \\
\hline TyG-BMI & $152.43 \pm 16.53$ & $169.94 \pm 18.38$ & $154.90 \pm 17.87$ & $\bigotimes 0.001$ \\
\hline TyG-WC & $569.38 \pm 65.76$ & $640.19 \pm 73.04$ & $579.38 \pm 71.24$ & $\varangle 0.001$ \\
\hline
\end{tabular}




\begin{tabular}{|c|c|c|c|c|}
\hline TyG-WHtR & $3.59 \pm 0.43$ & $4.04 \pm 0.48$ & $3.66 \pm 0.46$ & $\otimes 0.001$ \\
\hline \multicolumn{5}{|l|}{ Lifestyle } \\
\hline Current smoking & $3063(12.65)$ & $357(1.47)$ & $3420(14.12)$ & 0.078 \\
\hline Current drinking & $2962(12.23)$ & $458(1.89)$ & $3420(14.12)$ & $\varangle 0.001$ \\
\hline $\begin{array}{l}\text { Frequency of } \\
\text { physical activity }\end{array}$ & & & & 0.222 \\
\hline Low & $13,094(54.07)$ & $2140(8.84)$ & $15,234(62.91)$ & \\
\hline Moderate & 2993(12.36) & $529(2.18)$ & $3522(14.54)$ & \\
\hline High & $4708(19.44)$ & $751(3.10)$ & $5459(22.55)$ & \\
\hline
\end{tabular}

Abbreviations: T2DM,Type 2 Diabetes Mellitus; BMI, Body Mass Index; SBP, Systolic Blood Pressure, DBP, Diastolic Blood Pressure; TC, Total Cholesterol; TG, Triglyceride; HDL-C, high-density lipid cholesterol; LDL-C, low-density lipid cholesterol; FPG, fasting plasma glucose; WC, Waist Circumference; WHtR, Waist HeightRatio; VAI, Visceral Adiposity Index; LAP, Lipid Accumulation Product; TyG, Triglyceride Glucose; TyG-BMI, TyG related to BMl; TyG-WC, TyG related to WC; TyG-WHtR, TyG related to WHtR; Data are presented as means \pm standard deviation, and interquartile range or frequencies. $p$ values are from $t$-tests or Chi-square tests for analysis of variance for continuous variables and categorical variables

Table 2 aOR for T2DM in quartiles of each index 


\begin{tabular}{|c|c|c|c|c|}
\hline Categories & WC & WHtR & VAI & LAP \\
\hline \multicolumn{5}{|l|}{ Men } \\
\hline Q1 & Ref. & Ref. & Ref. & Ref. \\
\hline Q2 & $1.216(0.974,1.518)$ & $0.991(0.819,1.201)$ & $1.163(0.947,1.429)$ & $1.224(0.972,1.542)$ \\
\hline Q3 & $1.239(0.946,1.624)$ & $0.876(0.965,1.103)$ & $1.373(1.090,1.729)$ & $1.227(0.927,1.624)$ \\
\hline Q4 & $1.514(1.130,2.029)$ & $1.095(0.842,1.425)$ & $1.933(1.479,2.527)$ & $1.553(1.099,2.094)$ \\
\hline \multicolumn{5}{|l|}{ Women } \\
\hline Q1 & Ref. & Ref. & Ref. & Ref. \\
\hline Q2 & $1.123(0.955,1.320)$ & $0.879(0.732,1.056)$ & $1.155(0.965,1.382)$ & $1.315(1.088,1.589)$ \\
\hline Q3 & $1.085(0.890,1.323)$ & $0.952(0.781,1.159)$ & $1.567(1.278,1.920)$ & $1.428(1.136,1.793)$ \\
\hline Q4 & $1.133(0.908,1.414)$ & $0.989(0.789,1.240)$ & $1.954(1.541,2.478)$ & $1.754(1.337,2.300)$ \\
\hline Categories & TyG & TyG-BMI & TyG-WC & TyG-WHtR \\
\hline \multicolumn{5}{|l|}{ Men } \\
\hline Q1 & Ref. & Ref. & Ref. & Ref. \\
\hline Q2 & $3.136(2.226,4.339)$ & $0.966(0.735,1.270)$ & $1.122(0.785,1.604)$ & $1.593(1.166,2.177)$ \\
\hline Q3 & $6.819(4.867,9.545)$ & $0.783(0.585,1.048)$ & $0.976(0.641,1.487)$ & $1.572(1.080,2.089)$ \\
\hline Q4 & $19.005(13.235,27.291)$ & $0.968(0.705,1.329)$ & $1.122(0.705,1.786)$ & $2.356(1.557,3.566)$ \\
\hline \multicolumn{5}{|l|}{ Women } \\
\hline Q1 & Ref. & Ref. & Ref. & Ref. \\
\hline Q2 & $3.347(2.268,4.938)$ & $0.809(0.628,1.041)$ & $1.115(0.811,1.533)$ & $1.075(0.745,1.550)$ \\
\hline Q3 & 7.568(5.128,11.167) & $0.775(0.601,1.000)$ & $1.239(0.861,1.782)$ & $1.247(0.822,1.892)$ \\
\hline Q4 & $25.262(16.957,37.634)$ & $1.024(0.785,1.335)$ & $1.723(1.164,2.552)$ & $1.264(0.809,1.974)$ \\
\hline
\end{tabular}

Abbreviations: aOR, adjusted Odds Ratio; Q, Quartile; Ref, Reference; WC, Waist Circumference; WHtR, Waist Height-Ratio; VAI, Visceral Adiposity Index; LAP, Lipid Accumulation Product; TyG, Triglyceride Glucose; TyGBMI, TyG related to BMI; TyG-WC, TyG related to WC; TyG-WHtR, TyG related to WHtR;

Table 3 ROC curve analyses for each index in predicting T2DM 


\begin{tabular}{|c|c|c|c|c|}
\hline Parameters & wC & WHtR & VAI & LAP \\
\hline \multicolumn{5}{|l|}{ Man } \\
\hline $\operatorname{AUC}(95 \% \mathrm{Cl})$ & $0.586(0.570,0.602)$ & $0.571(0.555,0.587)$ & $0.620(0.604,0.636)$ & $0.622(0.606,0.638)$ \\
\hline$p$-value & $\otimes 0.001$ & $\llbracket 0.001$ & $\otimes 0.001$ & $\otimes 0.001$ \\
\hline Cut-off & $\geq 84.3$ & $\geq 0.49$ & $\geq 1.50$ & $\geq 25.08$ \\
\hline Sens. (\%) & 50.22 & 70.80 & 48.55 & 51.52 \\
\hline Spec. (\%) & 62.57 & 39.19 & 69.67 & 66.53 \\
\hline Youden index & 0.13 & 0.10 & 0.18 & 0.18 \\
\hline \multicolumn{5}{|l|}{ Women } \\
\hline $\operatorname{AUC}(95 \% \mathrm{Cl})$ & $0.565(0.552,0.578)$ & $0.556(0.543,0.570)$ & $0.625(0.612,0.638)$ & $0.630(0.617,0.643)$ \\
\hline$p$-value & $\llbracket 0.001$ & $\llbracket 0.001$ & $\otimes 0.001$ & $\otimes 0.001$ \\
\hline Cut-off & $\geq 79.8$ & $\geq 0.52$ & $\geq 2.04$ & $\geq 33.61$ \\
\hline Sens. (\%) & 67.16 & 65.03 & 60.29 & 58.38 \\
\hline Spec. (\%) & 42.70 & 42.97 & 59.26 & 60.80 \\
\hline Youden index & 0.10 & 0.09 & 0.19 & 0.19 \\
\hline Man & TyG & TyG-BMI & TyG-WC & TyG-WHtR \\
\hline AUC $(95 \% \mathrm{Cl})$ & $0.818(0.810,0.825)$ & $0.760(0.752,0.768)$ & $0.771(0.763,0.779)$ & $0.768(0.760,0.776)$ \\
\hline$p$-value & $\otimes 0.001$ & $\llbracket 0.001$ & $\llbracket 0.001$ & $\otimes 0.001$ \\
\hline Cut-off & $\geq 7.17$ & $\geq 163.04$ & $\geq 608.91$ & $\geq 3.64$ \\
\hline Sens. (\%) & 76.09 & 62.54 & 66.67 & 70.58 \\
\hline Spec. (\%) & 72.38 & 77.35 & 73.84 & 69.20 \\
\hline Youden index & 0.48 & 0.40 & 0.41 & 0.40 \\
\hline \multicolumn{5}{|l|}{ Women } \\
\hline $\operatorname{AUC}(95 \% \mathrm{Cl})$ & $0.824(0.814,0.833)$ & $0.760(0.748,0.771)$ & $0.766(0.755,0.777)$ & $0.760(0.749,0.771)$ \\
\hline$p$-value & $₫ 0.001$ & $\llbracket 0.001$ & $\llbracket 0.001$ & $\otimes 0.001$ \\
\hline Cut-off & $\geq 7.40$ & $\geq 162.68$ & $\geq 592.40$ & $\geq 3.88$ \\
\hline Sens. (\%) & 73.87 & 68.24 & 73.13 & 70.98 \\
\hline Spec. (\%) & 75.90 & 70.84 & 66.16 & 68.36 \\
\hline Youden index & 0.50 & 0.39 & 0.40 & 0.39 \\
\hline
\end{tabular}


Abbreviations: ROC, Receiver Operating Characteristic Curve; AUC, Area Under Curve; 95\% Cl, 95\% Confidence Interval; Sens, Sensitivity; Spec, Specificity; WC, Waist Circumference; WHtR, Waist Height-Ratio; VAl, Visceral Adiposity Index; LAP, Lipid Accumulation Product; TyG, Triglyceride Glucose; TyG-BMI, TyG related to BMl; TyG-WC, TyG related to WC; TyG-WHtR, TyG related to WHtR;

\section{Table 4 Pairwise comparison of AUC of TyG}

\begin{tabular}{|c|c|c|c|}
\hline Index & Differences between AUC & $95 \% \mathrm{Cl}$ & $p$-value \\
\hline \multicolumn{4}{|l|}{ Men } \\
\hline WC & 0.232 & $(0.213,0.250)$ & $\otimes 0.001$ \\
\hline WHtR & 0.246 & $(0.268,0.265)$ & $\varangle 0.001$ \\
\hline VAl & 0.198 & $(0.188,0.207)$ & $\varangle 0.001$ \\
\hline LAP & 0.195 & $(0.185,0.260)$ & $\varangle 0.001$ \\
\hline TyG-BMI & 0.056 & $(0.048,0.066)$ & $\bowtie 0.001$ \\
\hline TyG-WC & 0.046 & $(0.036,0.055)$ & $\otimes 0.001$ \\
\hline TyG-WHtR & 0.050 & $(0.040,0.059)$ & $\otimes 0.001$ \\
\hline \multicolumn{4}{|l|}{ Women } \\
\hline WC & 0.259 & $(0.243,0.274)$ & $\otimes 0.001$ \\
\hline WHtR & 0.268 & $(0.252,0.284)$ & $\otimes 0.001$ \\
\hline VAI & 0.199 & $(0.191,0.206)$ & $\otimes 0.001$ \\
\hline LAP & 0.194 & $(0.186,0.202)$ & $\varangle 0.001$ \\
\hline TyG-BMI & 0.064 & $(0.056,0.072)$ & $\bowtie 0.001$ \\
\hline TyG-WC & 0.058 & $(0.050,0.066)$ & $\varangle 0.001$ \\
\hline TyG-WHtR & 0.064 & $(0.055,0.073)$ & $\otimes 0.001$ \\
\hline
\end{tabular}

Abbreviations: AUC, Area Under Curve; 95\% Cl, 95\% Confidence Interval; WC, Waist Circumference; WHtR, Waist Height-Ratio; VAI, Visceral Adiposity Index; LAP, Lipid Accumulation Product; TyG, Triglyceride Glucose; TyG-BMI, TyG related to BMI; TyG-WC, TyG related to WC; TyG-WHtR, TyG related to WHtR;

\section{Figures}



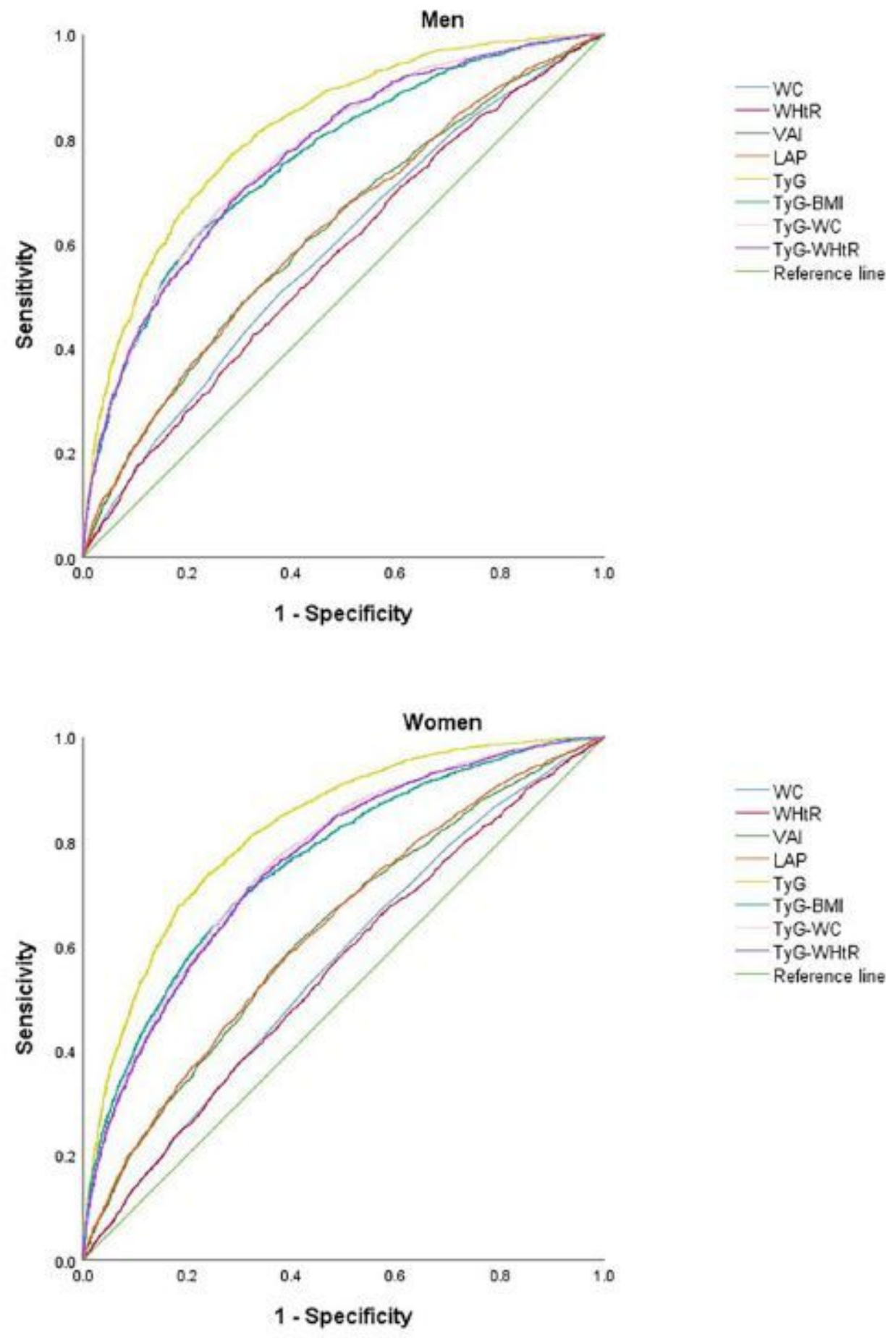

\section{Figure 1}

ROC curves for each indices as predictors of T2DM in men and women. 